\title{
Dorsal skin fold chamber for high resolution multiphoton imaging
}

\author{
FENG-CHIEH LI I , CHUN-CHIN WANG ${ }^{1}$, SUNG-JAN LIN ${ }^{2}$, \\ SHIOU-HWA JEE ${ }^{2,3}$, WEN LO ${ }^{1}$ AND CHEN-YUAN DONG ${ }^{1, *}$ \\ ${ }^{1}$ Department of Physics, National Taiwan University, Taipei 106, Taiwan, ROC \\ ${ }^{2}$ Department of Dermatology, National Taiwan University Hospital, Taipei 100, Taiwan, ROC \\ ${ }^{3}$ Department of Dermatology, College of Medicine, National Taiwan University Hospital, Taipei 100, \\ Taiwan, $R O C$ \\ (*author for correspondence: E-mail: cydong@phys.ntu.edu.tw)
}

Received 28 February 2005; accepted 15 September 2005

\begin{abstract}
The advantages offered by multiphoton microscopy enabled this technique to be applied for in vivo understanding of physiological processes. However, multiphoton intravital microscopy also requires associated technologies to be developed. In this work, we detailed the design of a dorsal skin fold chamber made of titanium alloy that allow high resolution multiphoton fluorescence and second harmonic generation (SHG) microscopy to be achieved. We demonstrate that our apparatus is capable of obtaining high resolution, images of blood vessels and collagen matrix in nude mice. Such an imaging chamber will allow physiological processes to be investigated at high resolution.
\end{abstract}

Key words: dorsal skin fold chamber, multiphoton imaging, second harmonic generation

\section{Introduction}

The introduction of two-photon fluorescence microscopy revolutionized bioimaging at the submicron level. A number of advantages are associated with this approach. First, the high photon flux required to induce the non-linear optical effects limit the sample excitation to the focal volume. Bioimaging achieved by scanning the point-like excitation volume results in images with excellent axial depth discrimination. In addition, the pointlike excitation volume also limits the specimen photodamage to the focal volume, thus prolonging sample longevity. Furthermore, the near-infrared photons commonly used to induce multiphoton processes in biological specimens are absorbed and scattered less than the ultraviolet or visible photons used in one-photon microscopy. As a result, greater sample imaging depths can be achieved without invasive histological procedures. Finally, the wide spectral separation between the near-infrared photons and specimen luminescence allows more efficient detection to be achieved (Denk et al. 1990; So et al. 2000). These advantages have allowed multiphoton microscopy to be widely applied to a number of diverse fields in 
biology and medicine such as neurobiology, developmental biology, immunology, cancer biology, hepatology, transdermal delivery, and deep tissue imaging. (Denk et al. 1995; So et al. 1998; Squirrel et al. 1999; Yu et al. 2001; Miller et al. 2002; Brown et al. 2003; Sun et al. 2003; Lee et al. 2004) In addition to multiphoton fluorescence imaging, the non-linear effect of SHG can also be utilized to probe biological structures such as the colla gen matrix. (Zoumi et al. 2002; Campagnola and Lowe 2003; Zipfel et al. 2003; Lin et al. 2005).

As multiphoton microscopy has been demonstrated to be a powerful imaging modality in biology, related technologies need to be developed in order to facilitate the extension of non-linear microscopy to in vivo studies. One example is the dorsal skin fold chamber which allows in vivo microscopy to be achieved in animals (Brown et al. 2003). In this work, we describe the design of a compact, lightweight, dorsal skin fold chamber that may be used to investigate in vivo physiological processes at high resolution. By combining the high resolution imaging chamber with multiphoton microscopy, we showed that we can image SHG collagen fibers and dye filled blood vessels inside nude mice. We envision this apparatus to be useful to probe a variety of physiological processes at high resolution.

\section{Materials and methods}

\subsection{MULTIPHOTON MICROSCOPE}

The multiphoton microscope used in this study is similar to a system described previously (Sun et al. 2003). A titanium-sapphire laser (Tsunami, Spectra Physics, Mountain View, CA) pumped by a diode-pumped solidstate laser (Millennia, Spectra Physics) was used as the excitation source. The near-infrared photons were guided toward a modified, commercial upright microscope (E800, Nikon, Japan) by an $x-y$ mirror scanning system (Model 6220, Cambridge Technology, Cambridge, MA). The entrance port of the microscope was modified to accommodate a beam expander. The beam expanded laser is then reflected into the back aperture of the high numerical aperture (NA) focusing objective (40X, S Fluor, NA 1.3, Nikon) by a dichroic mirror. The generated fluorescence and SHG signals were collected, respectively, in the epi-illuminated and back-scattering geometry by the same focusing objective. After passing through the dichroic mirror and additional filters, the spectrally resolved luminescence from specimen are detected by photomultiplier tubes (R7400P, Hamamatsu, Japan). The single-photon counting approach was used to process the signal photons. In 
the case that a large area multiphoton image is desired, a sample position stage (H101, Prior Scientific, UK) was used to translate the specimen after each small area multiphoton scan.

\subsection{HIGH RESOLUTION DORSAL SKIN FOLD CHAMBER}

Central to this study is the design of the high resolution dorsal skin fold chamber. To ensure compatibility with the mouse model animal, we employed titanium alloy (6AL/4V ELI) in the chamber construction. As Fig. 1 shows, the chamber is composed of three main components: a front

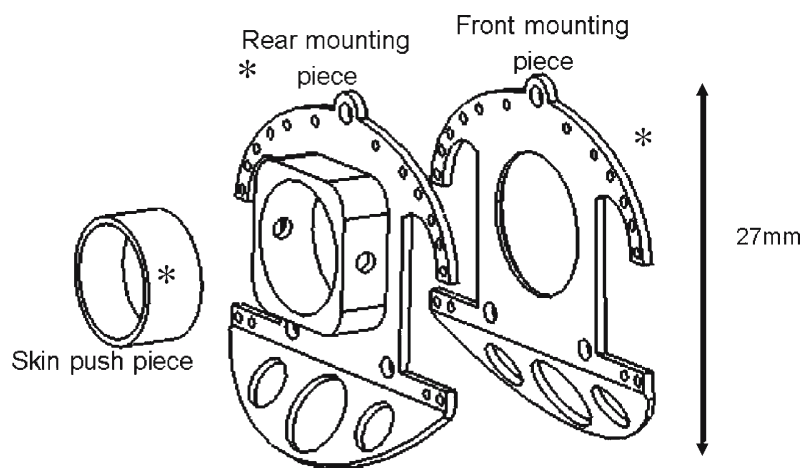

Fig. 1. The three main components of the high resolution dorsal skin fold chamber.

Front mounting piece

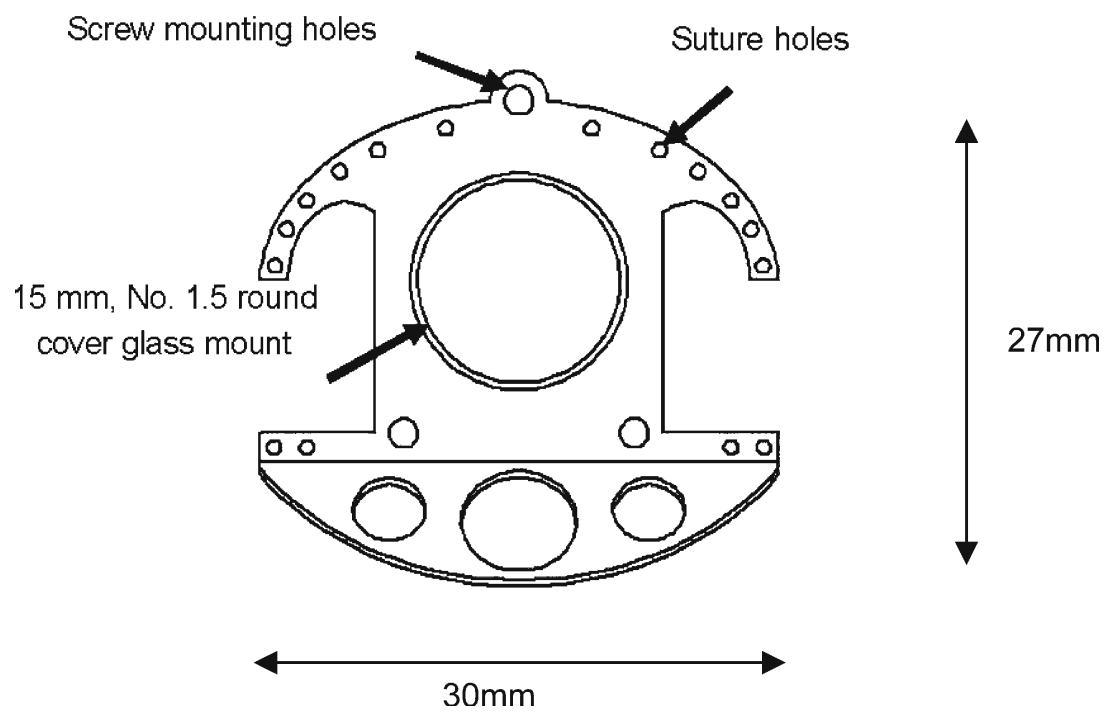

Fig. 2. Detailed design of the front mounting piece. 
mounting piece, a rear mounting piece, and a skin push piece. The basic design principle of the chamber system is to sandwich the skin of the animal model in between the front and rear mounting pieces. As shown in Fig. 2, a groove exists in the front mounting piece to allow a $15 \mathrm{~mm}$ round cover glass to be mounted as the viewing window into the animal model. In our studies, we use the standard No. 1.5 cover glasses for viewing purposes. The use of No. 1.5 cover glasses ensures the ability to use high NA objectives for high resolution imaging. In both the front and rear mounting pieces, a series of small suture holes exist along the edges. In addition, three larger screw mounting holes were implemented into the upper portion of the mounting pieces. The suture holes are designed to suture the skin onto the mounting pieces while the purpose of the screw mounting holes is to add additional support to secure the two mounting pieces and the skin into an integral unit. The screw mounting holes were designed to allow the use of M2 nuts and screws for mounting purposes. In our studies, once the two main mounting pieces are securely attached to the skin and the cover glass mounted, the skin push piece can be used to push against the skin into firm physical contact with the front cover glass to allow high resolution microscopy to be performed. Additional measures were made to minimize the stress of the chamber on the model animal. First, the bottoms of the two main mounting pieces were bent to create a saddle (Fig. 1). In addition, to reduce the extra weights, the main pieces are made to be $1 \mathrm{~mm}$ thick and the interior of the set screw mounted skin push piece was bored. The approximate weight of the entire high resolution skin fold chamber is approximately $5.5 \mathrm{~g}$. At approximately $18 \%$ the body mass of the nude mouse chosen for our study, the chamber appears not to have significantly altered the mobility or the daily life of animal model.

\subsection{APPLYING THE HIGH RESOLUTION DORSAL SKIN FOLD CHAMBER FOR MULTIPHOTON IMAGING}

The high resolution dorsal skin fold chamber was designed to be applied for multiphoton imaging into BALB/c-nu mice. These nude mice were chosen as the lack of skin hair eases the chamber mounting procedures. To mount the chamber onto the mouse, anesthesia was applied to the animal. A stock solution of pentobarbital in phosphate buffered saline (PBS) at the concentration of $20 \mathrm{mg} / \mathrm{ml}$ was prepared. $50 \mu \mathrm{l}$ of the stock anesthesia solution was injected intraperitoneally.

After anesthesia and wiping of the mouse with aqueous ethanol, the rear mounting piece was sutured onto the mouse. After a period on the order of days, the front mounting piece is also sutured onto the skin and the 
mounting screws attached. The chamber components were immersed in the aqueous ethanol to minimize possible infections. After both the mounting pieces were attached, the skin exposed at the front mounting piece was partially removed. Finally, the No. 1.5 cover glass is mounted and secured with nail polish.

In imaging the mouse, we employed SHG intensity to probe local collagen structures and fluorescence to image the vascular network. To image the vascular network inside the mouse, rhodamine B isothiocyanate-dextran (MW70,000, Sigma, St. Louis, MO) was employed. The rhodamine Bdextran imaging solution was prepared by generating a stock solution at the concentration of $50 \mathrm{mg} / \mathrm{ml}$ and $100 \mu \mathrm{l}$ of the stock solution was injected through the mouse tail.

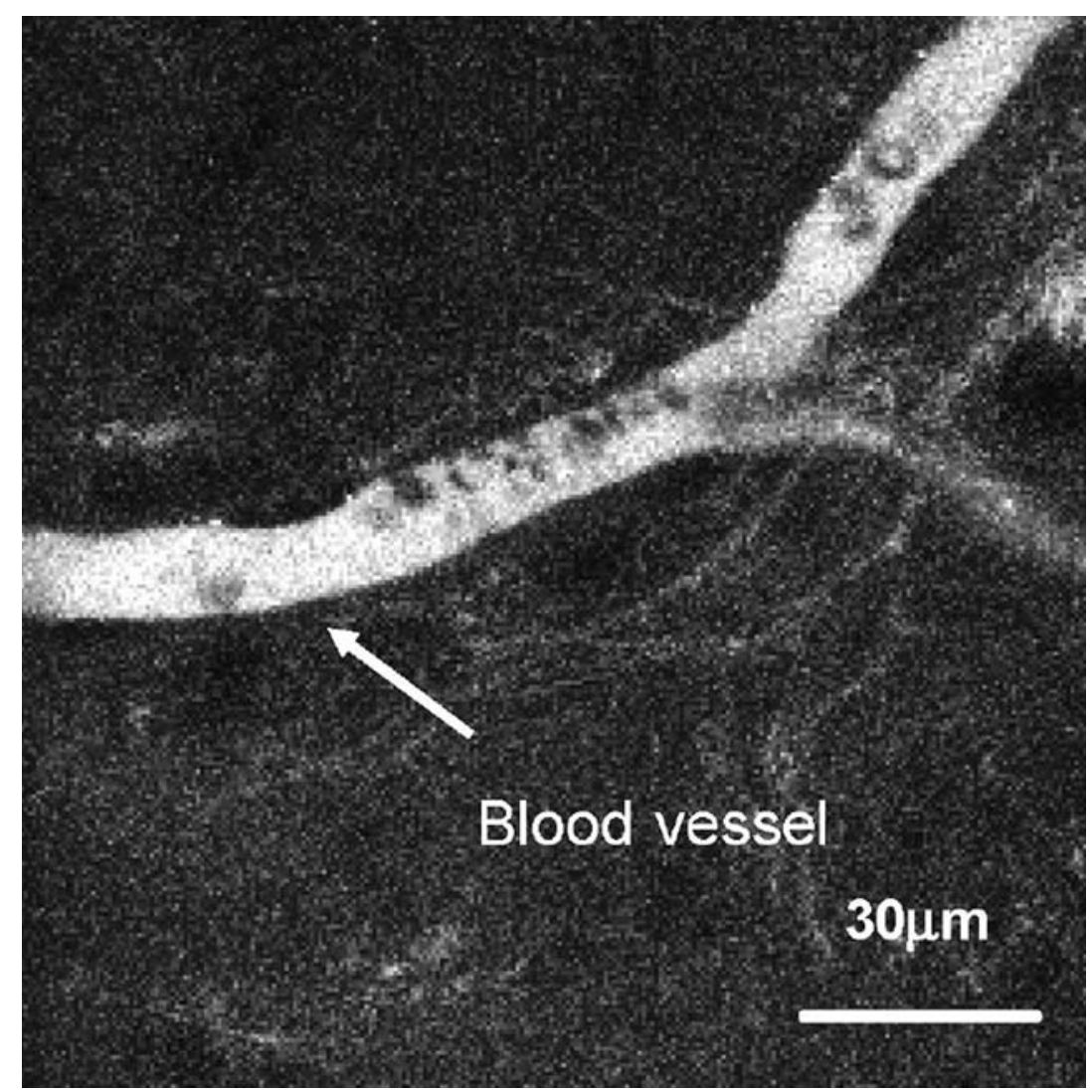

Fig. 3. High resolution multiphoton fluorescence image of the rhodamine-dextran filled blood vessel. 


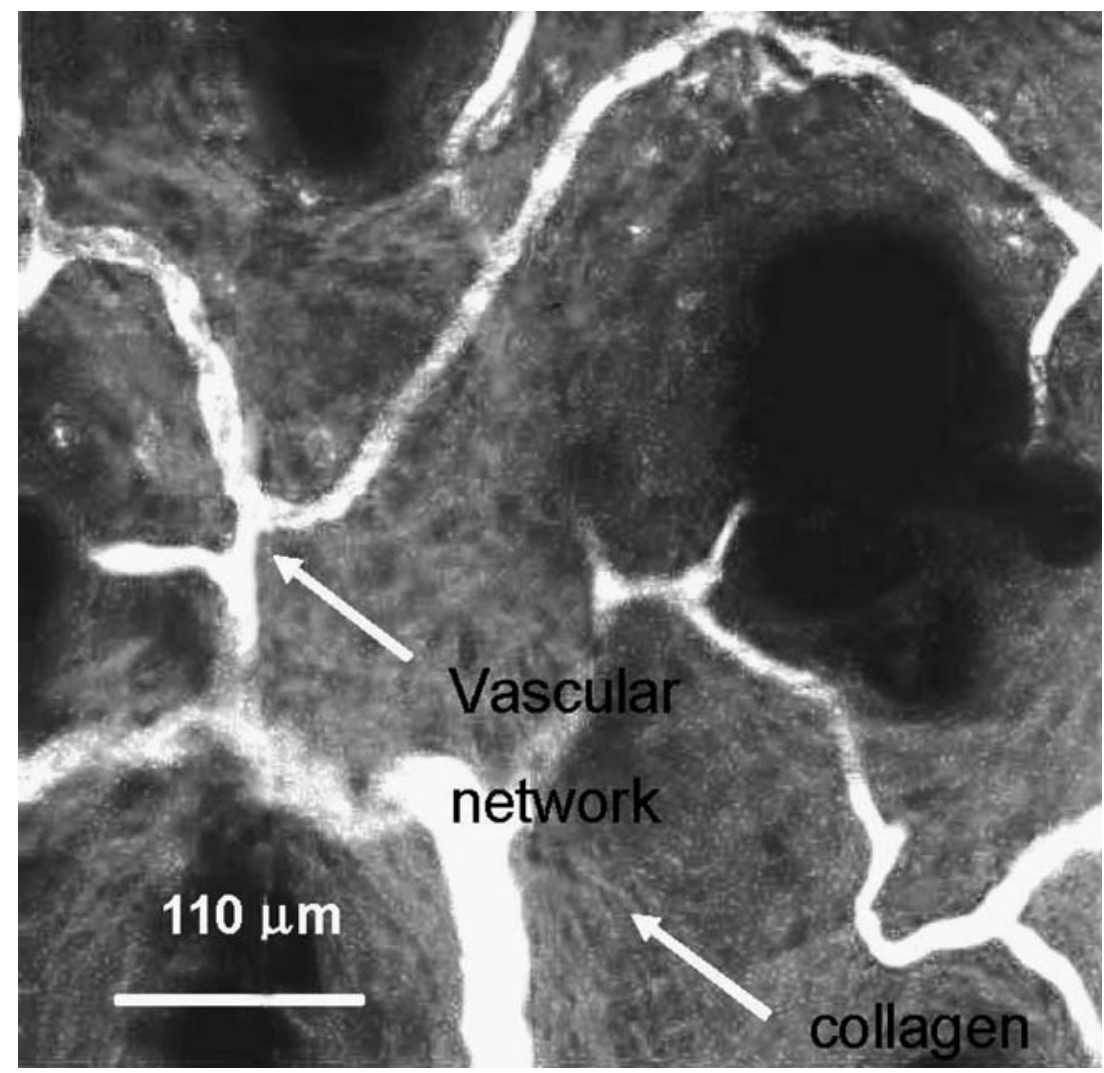

Fig. 4. Multiphoton fluorescence (vascular network) and SHG (collagen) within the mouse.

\section{Vascular and collagen imaging}

To demonstrate the application of our imaging chamber design, we have performed multiphoton microscopy to acquire the rhodamine B-dextran filled blood vessel (Fig. 3). In Fig. 4, we extended our demonstration by performing a large area imaging of both the vascular network and local organization of the collagen matrix by fluorescence and SHG microscopy, respectively. In all the images, high resolution was easily achieved. To our surprise, the thin No. 1.5 cover glass can be mounted on the order of days without being broken.

\section{Conclusions}

We have demonstrated the successful design and implementation of a dorsal skin fold chamber for high resolution multiphoton microscopy. We have 
shown that high resolution microscopy of the vascular structure and collagen arrangement can be imaged using a combination of fluorescence and SHG microscopy. As multiphoton microscopy is a powerful imaging modality for probing the physiological processes in vivo, we envision the developments of technologies similar to the one described in this work to be invaluable additions in achieving intravital multiphoton imaging.

\section{Acknowledgement}

We acknowledge the support of NSC 93-3112-B-002-033 (National Science Council, Taiwan, ROC) for the completion of this project. Feng-Chieh Li and Chun-Chin Wang contributed equally to this work.

\section{References}

Brown, E., T. McKee, E. diTomaso, A. Pluen, B. Seed, Y. Boucher and R.K. Jain. Nat Med. 9, 796, 2003.

Campagnola, P.J. and L.M. Loew. Nat. Biotechnol. 21 1356, 2003.

Denk, W., M. Sugimori and R. Llinas. PNAS 92 8279, 1995.

Denk, W., J.H. Strickler and W.W. Webb. Science 248(4951) 73, 1990.

Lee H.S., Y. Liu, H.C. Chen, L.L. Chiou, G.T. Huang, W. Lo and C.Y. Dong. Opt Lett. 29 2614, 2004. Lin, S.J., C.Y. Hsiao, Y. Sun, W. Lo, W.C. Lin, G.J. Jan, S.H. Jee and C.Y. Dong. Opt. Lett. 30(6) 622, 2005.

Miller, M. J., S. H. Wei, I. Parker and M.D. Cahalan. Science 296 1869, 2002.

So, P.T.C., C.Y. Dong, B.R. Masters and K.M. Berland. Annu. Rev. Biomed. Eng. 2 399, 2000.

So, P.T.C., H. Kim and I.E. Kochevar. Opt. Expr. 3 339, 1998.

Squirrell, J.M., D.L. Wokosin, J.G. White and B.D. Bavister. Nat. Biotechnol. 17 763, 1999.

Sun, Y., W. Lo, S.J. Lin, S.H. Jee and C. Y. Dong. Opt. Lett. 29 2013, 2004.

Sun, Y., J.W. Su, W. Lo, S.J. Lin, S.H. Jee and C.Y. Dong. Opt. Expr. 11(25) 3377, 2003.

Yu B., C.Y. Dong, P.T.C. So, D. Blankschtein and R. Langer. J. Invest. Dermatol. 117 16, 2001

Zipfel, W.R., R.M. Williams, R. Christie, A.Y. Nikitin, B.T. Hyman and W. W. Webb. PNAS 1007075 , 2003.

Zoumi, A., A. Yeh and B.J. Tromberg. PNAS 99 11014, 2002. 